\title{
Fast Tomography Imaging System for Material Surface Based on Doublet-cylinder-lens in Intelligent OCT
}

\author{
Shijun $\mathrm{Xu}^{1, \mathrm{a}}$ and Xiaoling Ren ${ }^{2,3, \mathrm{~b}}$ \\ ${ }^{1}$ School of Science, Xi'an Technological University, Xi' an 710021, China \\ ${ }^{2}$ School of Computer, Xi'an Polytechnic University, Xi'an 710048, China \\ ${ }^{3}$ Department of Materials Science, University of Delaware, Newark, DE 19716, USA \\ axushijun000@sina.com, ${ }^{\mathrm{b}}$ renxiaoling2@163.com
}

\begin{abstract}
A fast lateral line-scan mode in OCT was investigated. The elimination of spherical aberration, the beam mode and the focus of doublet-cylinder-lens, were theoretically analyzed. The parameters and signal characteristics of the confocus microscopy system with doublet-cylinder-lens were analyzed and simulated. The relation between the transverse resolution and the numerical aperture and the depth of focus (DOF) was discussed in the OCT. Our results show that the spherical aberration and the sine aberration are effectively eliminated in the doublet-cylinder-lens with K9-ZF2 glass. The beam is uniformly focused on the arc-sagitta-plane of cylinder lens, and the parallel interference pattern with axial symmetry is in favor of improving transverse resolution. The transverse resolution depends upon the numerical aperture and the DOF. The scanning velocity of doublet-cylinder-lens confocus microscopy system is 103 times faster than the traditional spot-scan, while the transverse resolution is $15 \mu \mathrm{m}$ and the constraints of aperture $50.8 \mathrm{~mm}$. This advanced system will be valuable in real-time imaging to material surface.

Keywords. Material surface testing, optical coherence tomography (OCT), confocus microscopy, line-scan, doublet-cylinder-lens
\end{abstract}

\section{Introduction}

For optical coherence tomography system (OCT) on testing material surface, main restriction or shortcoming focus on the imaging velocity of the scanning process, which complexity inclued two parts called axial scanning and transverse scanning. Thus, many solutions to solve this problem has been explored. Specifically, one way was to enhance the velocity of axial scanning by application of different scanning devices [1]. Although the velocity could be boosted, the demand for high speed, stability and accuracy may cause very high investment. Another way was proposed in 2000 by Bourquin etc, which could achieve the parallel OCT of video rates (Parallel OCT) [2]. But this full-range detecting technology scanning has a very small range, so it need the intelligent CMOS image sensor, the lack of communication mode makes it vulnerable to interferences made by different $1 / f$ noise sources, as well as lack of narrowband heterodyne demodulation technology makes interferences from noises relying on bandwidth intensified.

Line focus is proposed in 1999 by Suekuni etc. researching about spectrum OCT scanning mode. This way can get optical path difference directly through the Fourier transform and spectral density function [3-5]. This line scanning mode is similar with scanning mode, mainly has expansion interms of the transverse scan and reduced the cycle of transverse scan process. The use of slit in the expanded way of light decreased the flux while restricting the shape of the beam, thus reduced the efficiency of light source use, therefore, and caused less depth imaging and low SNR.

In our study, a rectangular aperture is used behind the expanded beam, and we obtain line focus with a cylinder lens. To reducing the scanning time, samples are linearly scanned through the cylinder lens of focal microscopy system. This line focusing model could transfer horizontal point scanning into parallel scanning mode, simplified the scanning process, and the shortened the scanning time. In the mean time, it can also retain the advantages of point scanning, which can effectively restrain the stray light with the confocal scanning. The time for getting $3 \mathrm{D}$ chromatography image could be shortened by three orders of magnitude. 


\section{Drawbacks of OCT with Point-focus-scan Mode}

In the line scanning of OCT with point-focus-scan mode, the light beam going through the beam splitter is focused to a point via focusing lens. Light focus point will stay or suspend on each scan position of the sample. Finished the axial scanning, it will be moved to the next position by the line scanning device, and then going on the axial scanning. Firstly, one keeps $\mathrm{Y}$ direction invariable and conducts point scanning along the $\mathrm{X}$ direction. Secondly, the light focus point is moved to the next row of $\mathrm{Y}$ direction. Finally, the above point scanning along the $\mathrm{X}$ direction is repeated. Obviously, the repeating scanning along the $\mathrm{X} \& \mathrm{Y}$ point by point, which is "static-accelerate-slow-static" mode, will exhaust much time [1-2].

\section{Line Scanning Structure and Characteristic}

In the general OCT system, after expanding the source light beam we set a rectangular or square aperture in front of the expanding beam, and put a confocus microscopy system with double-cylinder-lens near the sample. If we use the linear CCD or CMOS detector, which match with the double-cylinder-lens, a rapid transverse line scanning should be realized.

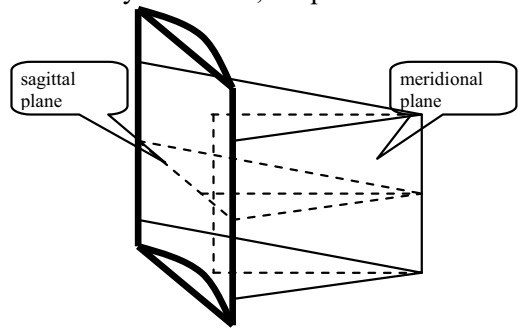

Fig.1. Imaging mode of cylinder lens.

The Beam Mode and the Focus of Doublet-cylinder-lens. As shown in the figure1, to show the three-dimensional light beam form, we plot two mutually perpendicular planes, one plane parallels to the beam axis and contains the interface normals (meridional plane), the other plane also parallels to the beam axis and is perpendicular to the meridional plane, that is to say a sagittal plane. The characteristics of the cylindrical lens are as follows: the beam focus on the sagittal plane with the lens, and the light parallelling the meridional plane will not fracture. Therefore, these characteristics not only realize the line focusing but also can ensure the same strength on every point of the focusing line in theory.

The focal length of the cylindrical lens can be calculated according to the formula of the thin lens

$$
f=r_{1} r_{2} /(n-1)\left(r_{2}-r_{1}\right)
$$

where $n$ represents refractive index of single lens, $r_{1}$ and $r_{2}$ represent two spherical radiuses respectively. As the flat convex cylinder lens, Eq.(1) can be simplified as follows

$$
f=r_{1} /(n-1)
$$

Common Spherical Aberration of the Cylindrical Lens. Generally, spherical aberration appears in imaging process of the optical systems. A monochromatic light beam from a point on the axis goes through an optical system, if the beam with different apertures focuses on different positions of the axis, there will be a dispersion light spot near the image point, which is named spherical aberration of the imaging system. Therefore, spherical aberration is the optical aberration from an imaging system to axial object points with monochromatic and wide (in diameter) beam.

The junior spherical aberration to cylindrical lens is

$$
\delta L^{\prime}=-\frac{h^{4}}{2 n_{1} u_{1}^{\prime 2}}\left\{\frac{n+2}{n} \phi \rho^{2} \cdot \frac{2 n+1}{n-1} \phi^{2} \rho+\frac{n^{2}}{(n-1)^{2}} \phi^{3}\right\}
$$

If the lens are exposed to the air, then $n_{1}=1, h=l \times u$ in which $l$ is object distance, $u$ is light aperture angle, $h$ is beam height content in normal section of the object space. Where $u_{1}$ 'represents aperture angle of the image space, $\varphi$ is light focal power of lens, $\rho$ is lens curvature of the first surface. According to Eq.(3), when $\mathrm{d} \delta L^{\prime} / \mathrm{d} \rho=0$, we obtain the minimum of spherical aberration as follows

$$
\delta L_{0}^{\prime}=\frac{n(4 n-1)}{8(n-1)^{2}(n+2)} h^{2} \varphi
$$

As per Eq.(4), the spherical aberration can not be eliminated with single thin lens.

Corresponding to thin lens group, the junior spherical aberration [6] is

$$
\delta L^{\prime}=-\frac{h^{4}}{2 n_{1} u^{\prime 2}}\left[A_{1}\left(n_{1}, \varphi_{1}, \rho_{1}, \sigma_{1}\right)+A_{2}\left(n_{2}, \varphi_{2}, \rho_{2}, \sigma_{2}\right)\right]=-\frac{h^{4}}{2 n_{1} u_{1}^{\prime 2}}\left\{\frac{n+2}{n} \varphi \rho^{2} \cdot \frac{2 n+1}{n-1} \varphi^{2} \rho+\frac{n^{2}}{(n-1)^{2}} \varphi^{3}\right\}
$$


Elimination of Spherical Aberration of Doublet-cylinder-lens. For Spherical aberration correction, there are three methods: (1) Use diaphragm to cover part of the light through the edge of lens, thus to reduce the aperture angle, though simple, this method can narrow the optical diameter of the lens and reduce the intensity of illumination on image plane; (2) Via integration of two curved surface of the lens will become appropriate aspheric surface, which can eliminate the Spherical aberration, but the treating of non-spherical surfaces are very difficult; (3) applications of double glued structures or double separation and reunion structures can eliminate the Spherical aberration, so in order to eliminate the Spherical aberration, the doublet-cylinder-lens has a lot of potential.

However, no matter what kind of optical system we use, we can only eliminate the Spherical aberration caused by paraxial rays, but not the Spherical aberration caused by light of all aperture angles, so still remains the spherical aberration. Application of he doublet-cylinder-lens could solve this problem efficiently, specific explanation s are as follow.

Because plus lens often generate positive spherical aberration while concave lens often generate minus spherical aberration, the doublet-cylinder-lens system can be considered as optical system which was made up by plus lens and concave lens, which have three refraction surfaces. In generally, one makes the lens curvature $\rho_{1}$ of glued surface as variable, and chooses the glasses pair as K9+ZF2. Relevant parameters are as follows: K9 glass $n_{\mathrm{D}}=1.51637, v=64.1$, $n_{\mathrm{F}}=1.52196, n_{\mathrm{c}}=1.51389 ; \mathrm{ZF} 2$ glass $n_{\mathrm{D}}=1.67268, v=32.2, n_{\mathrm{F}}=1.68747, n_{\mathrm{c}}=1.66662$. Where $v$ is Abbe number. Substituting all the parameters into Eq.(5) and setting $\delta L^{\prime}=0$, we get

$$
\delta L_{0}^{\prime}=-\left(19090 \rho_{1}^{2}+871.33 \rho_{1}+9.943\right)=0
$$

The discriminant of Eq.(6) is less than zero (the discriminant is very small), so that the solution $\rho_{1}$ can be considered as the solution of the elimination of spherical aberration. According to calculation $\rho_{1}=-0.02282 / \mathrm{mm}, r_{2}=-43.82 \mathrm{~mm}$, we get the sine aberration $\mathrm{OSC}=0.00049$. The $\mathrm{OSC}$ is very small, thus it indicates that the spherical aberration and the sine aberration could be corrected in the same time.

Using the junior spherical aberration Eq. (6) of doublet-cylinder-lens and Matlab software, we get three sorts of curve about spherical aberration respectively (in figure 2). Figure 2(a) is the spherical aberration curve with Full Correction method, the curve demonstrates that other rays all have certain spherical aberration, except for the paraxial rays and the edge rays. Figure 2(b) is the spherical aberration curve with Over Correction method. Figure 2(c) is the spherical aberration curve with Under Correction method.

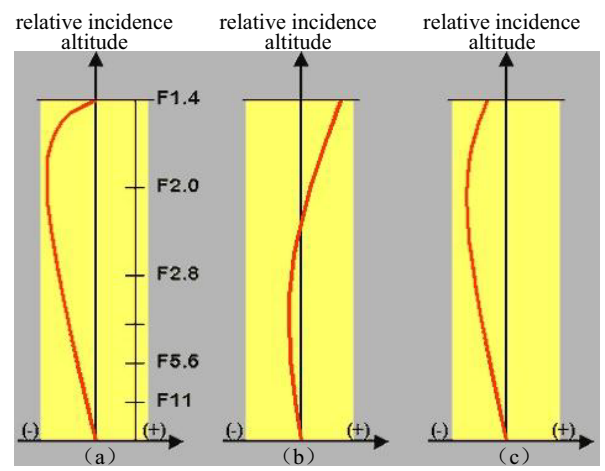

Fig.2. Spherical aberration curve of doublet-cylinder-lens.

Focal Length of Doublet-cylinder-lens. Considering the correction of spherical aberration and sine sent in transverse scan of the samples, doublet-cylinder-lens was used. Its focal length can be thought of the focal length as two thin lens joint together, whose focal length of the negative lens is

Whereas the focal length of the doublet-cylinder-lens is

$$
\begin{gathered}
f_{2}=r_{1} r_{2} /(n-1)\left(r_{2}-r_{1}\right) \\
f_{\text {double }}=r_{1} r_{2} /\left[r_{1}\left(n_{2}-1\right)-r_{2}\left(n_{1}+n_{2}\right)\right]
\end{gathered}
$$

where $n_{1}$ is the refractive index of the plus lens, while $n_{2}$ is the refractive index of the negative lens.

Confocus Microcopy with Doublet-cylinder-lens. In the scanning process of using confocus microcopy system with doublet-cylinder-lens, the stray light was greatly eliminated, the surrounding fluorescence generated background and stray light interference signals was restrained, so, this system has the gateway role ${ }^{[4,7]}$. The light outside of the objective lens focal plane was blocked, the signal-to-noise ratio of the probe got greatly improved, so the imaging surface has a very high contrast and clarity, and also can the system provide a high resolution. 


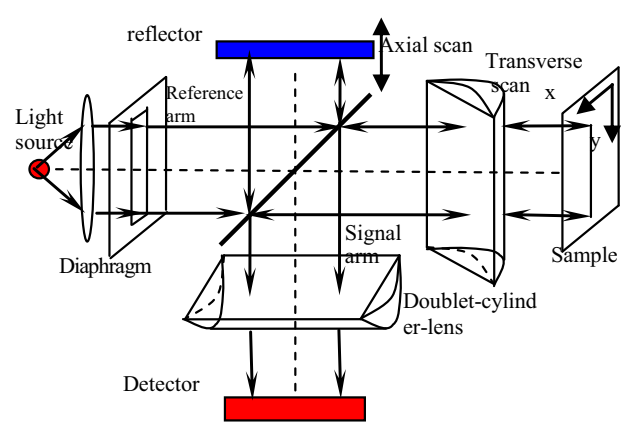

Fig. 3. OCT system with doublet-cylinder-lens.

Signal Characteristics. Fig.3 is the sketch of the OCT system with doublet-cylinder-lens. The light vector complex amplitude of the light from the light source can be expressed as

$$
E=A_{0} \exp (\mathrm{i} \varphi)
$$

After beam splitter, the light was divided into two beams, the first reflected by the beam splitter, then through the reflector, light vector of reference ray is

$$
E_{\mathrm{r}}=2^{(-1 / 2)} A_{0} \exp \left[\mathrm{i}\left(\varphi+k l_{\mathrm{r}}\right)\right]
$$

where $k$ is the wave vector. The second beam goes through the splitter, then it is focused by the doublet-cylinder-lens and projected to the surface and inner of the sample. The optical path differences caused by different focal points vary with different space position. So that the light vector distribution from the sample arm is

$$
E_{\mathrm{s}}=2^{(-1 / 2)} A_{0}{ }^{\prime} \exp \left[\mathrm{i}\left(\varphi+k l_{\mathrm{s}}\right)\right]
$$

where $l_{\mathrm{s}}=l_{\mathrm{s}}(y)$ is the optical length of the sample arm, $A_{0}{ }^{\prime}$ is the amplitude of the signal light modulated by the sample. After line focused by the cylinder lens, signal light reflected by samples and reference light reflected back by reflection lens were received by the detector. The overall interference light vector is

$$
E=E_{\mathrm{r}}+E_{\mathrm{s}}
$$

Assume $I_{0}=A^{2} / 4, I^{\prime}=A^{\prime 2} / 4$ ( $A$ and $A^{\prime}$ are considerated with loss of light intensity caused by the components like the doublet-cylinder-lens), then the stacked light intensity by two waves is

$$
I=\left\langle E E^{*}\right\rangle=I_{0}+I^{\prime}+2 \sqrt{I_{0} I^{\prime}} \cos k\left[l_{r}-l_{s}(y)\right]
$$

Let $\Delta=l_{\mathrm{r}}-l_{\mathrm{s}}(y)$, if $I_{0}=I^{\prime}$, we obtain

$$
I=2 I_{0}(1+\cos k \Delta)
$$

According to the type above, changes in the level of interference is still relevant with optical path difference. Because of the waken coherent of the light sources, the only reflected light or scattering light from the sample with a particular depth can coherent with the reference beam. The reflected lights from different depth of the samples have different phase delay, along with the increase of delay, interference signals fell sharply. The interference signals contain information of the sample, Therefore, after filtration and amplification we can get images of the samples.

In addition, the confocus microscopy system can increase the resolution of the system. Because the signal strength from outside of the focus is much weaker than within, and can greatly reduce the unwanted flickering noise and the noise from the scattering light.

System Signal Simulation. In the simulation to the above optical system, we adopted the ZEMAX from the INFOTEK corporate with monochromatic light (the wavelength is $1310 \mathrm{~nm}$ ). 


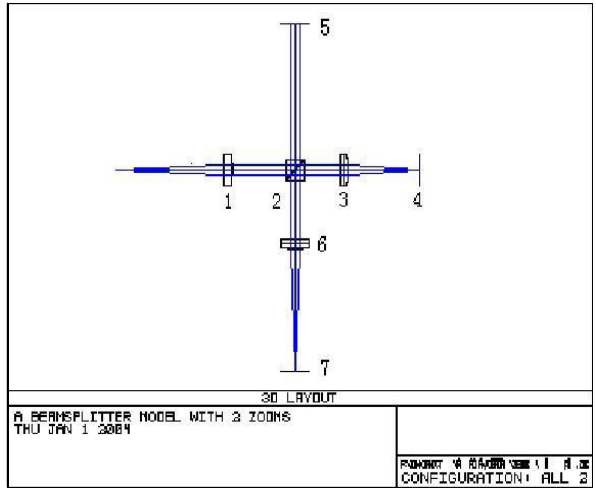

Fig.4. Simulation of the OCT system.

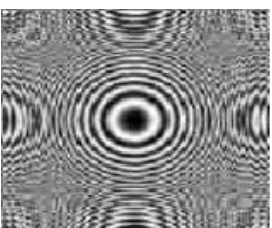

(a)

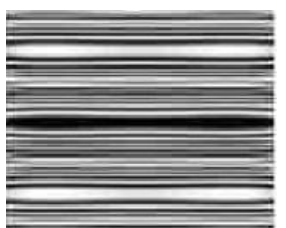

(b)

Fig.5. Two kinds of interference fringe.

The point lamp or light source are expanded to a collimator beam with a single lens. As shown in figure 4 of 3D shape of the main light through the center, component 1 is expanding lens, component 2 is the beam spliter, 3 and 6 are the doublet-cylinder-lens, 4 and 5 the plane mirror, 7 the imaging surface or detector, the light path contains plane mirror 5 is the reference arm, the light path contains the doublet-cylinder-lens 3 is the sample arm. In designing the length for the reference arm, we have already compensated the light path difference caused by cylinder mirror, thus the light paths on two arms is equal.

Simulation experiments are divided into two kinds of cases. One is thin round beam interference patterns on Michelson Interferometer, the simulation results showed in figure 5(a). The other simulation results are as shown in figure $5(\mathrm{~b})$, with the doublet-cylinder-lens, as the same specification in the light path of the sample arm, as in front of the detecting surface, and as the compensate for the optical path difference caused by them.

In figure 5(a) the interference patterns are concentric circles, while in figure 5(b) they are parallel interference plan stripes, indicates that under the limit of beam, the cylindrical lens are the same in terms of focusing light, namely on the line of focus, every points have the same optical path difference. This kind of axial symmetry parallel interference patterns are beneficial to enhance the Transverse resolution of the line scanning OCT system.

\section{Resolution Ratio and Focal Depth of Doublet-cylinder-lens Microscopy System.}

Theoretical Formula of the Resolution Ratio and Focal Depth. The adoption of doublet-cylinder-lens line scanning confocus system has the same axial resolution with point scan of OCT system, and they are all dependent on the coherent length of the light sources [8]

where $\lambda$ is the centre wavelength, $\Delta \lambda_{\mathrm{FWHM}}$ is the spectrum width of the light source.

$$
\Delta z_{F W H M}=\frac{2 \ln 2}{\pi} \cdot \frac{\lambda^{2}}{\Delta \lambda_{F W H M}}
$$

The horizontal resolution $\Delta x$ do not simply depend on the numerical aperture(NA) of the beam, it is also relevant with the distances between image sensitive units on the detector. The horizontal resolution of this doublet-cylinder-lens confocus microcopy system can also be shown as Eq. (16)

$$
\Delta x=\frac{4 \lambda}{\pi} \cdot \frac{f}{d}=\frac{4 \lambda r_{1} r_{2}}{\pi d\left[r_{1}\left(n_{2}-1\right)-r_{2}\left(n_{1}+n_{2}\right)\right]}
$$

where $d$ is the caliber of the parallel beams of the arm (and a square aperture caliber consistent).

If the calculated transverse resolution $\Delta x$ is greater than the distance of all probe light-sensing units. The transverse resolution of the line OCT scanning system is the value of $\Delta x$; If the calculated transverse resolution $\Delta x$ is smaller than the distance, the transverse resolution of the line OCT scanning system is the size of the light-sensing units. Obviously, the 
transverse resolution of the line OCT scanning system can not be higher than the size of the linear array CCD light-sensing units.

For the line scanning OCT system, Its focal depth (DOF) was decided by two times beam parameters of the confocus Gaussian beams, using Eq.(16), we have

$$
b=2 Z_{\mathrm{R}}=(\pi / 2)\left(\Delta x^{2} / \lambda\right)
$$

Relationship between Transverse Resolution \& NA \& Focal Depth. When increasing the transverse resolution, the gathering depth will decrease, which is similar with the nature and optical microscope. When using low numerical aperture, we can get great converged depth, but at this time the converge radius of the converging beams are bigger, so the transverse resolution will be lower; And when the system using high numerical aperture, the beam waist radius of converge beams will be lower at this time, which will increase the transverse resolution, but decrease the Confocal parameters, thus to decrease the converging depth.

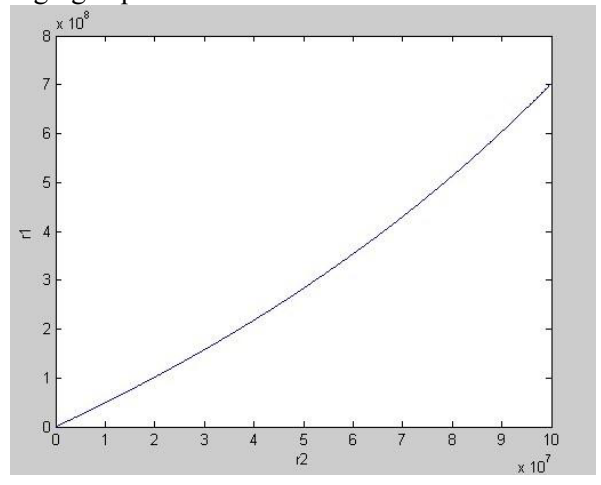

Fig.6. Relationship of $r_{1} \& r_{2}$ in doublet-cylinder-lens.

In order to improve the scanning speed, the wider width of the line scanning is the better. But in fact, the line width is not unlimited of increase. We should make optimization for those parameters. (1) On the scanning line, energy reduce of single point will cause the whole detecting ability to reduce; (2) To increase the line width, it have to increase beam diameter after beam expanding, this will also increase the numerical aperture, and decrease the focal depth; (3) The lager the line width, the more stray light on every detecting point, These stray light will interact with backscatter light from other points, which will reduce the signal-to-noise ratio(NSR), and affect the quality of scanning.

The parameters designed for this work are as follows: choosing the light center wavelength $1310 \mathrm{~nm}$, the caliber of the parallel beams with the sample arm for $\mathrm{d} 50.8 \mathrm{~mm}$. For glasses pairs, taking $K 9+Z F 2$ (above parameters have be given). As shown in figure 6 , when the transverse resolution is $15 \mu \mathrm{m}$, using Matlab software, we obtained the relationship between $r_{1}$ and $r_{2}$. When the axial resolution is $15 \mu \mathrm{m}$, we know the bandwidth $\Delta \lambda_{F W H M}$ is $50.5 \mathrm{~nm}$ according to Eq. (15).

\section{Summary}

The doublet-cylinder-lens not only inherited advantages of the traditional OCT system as its depth as point scanning and independent transverse scan resolution but can also Make up faults for traditional point scanning OCT technology, as to change the point scanning into line scanning, and the transverse scan resolution is also increased.

The main advantage of using confocus scanning mode is the flitting of the out focusing background. By the spherical aberration analysis for the cylindrical lens, and by test and verify via K9+ZF2 glasses, it's clear that this method can effectively eliminate the spherical aberration and the sine aberration. After analysis about the relationship between $r_{1}$ and $r_{2}$, we design the specific parameters for the doublet-cylinder-lens system. But because the line scan will distract energy of the detecting light, its detecting depth and signal-to-noise ratio will decrease with the increase of the line length, so, detectors with high sensitivity of detecting is essential.

Under the aperture $50.8 \mathrm{~mm} \times 50.8 \mathrm{~mm}$, the horizontal resolution is equal to $15 \mu \mathrm{m}$. In comparison with the point scanning mode under the same conditions, the new scanning speed at least achieves $3387(50.8 \mathrm{~mm} / 15 \mu \mathrm{m})$ times than that of single point scanning mode, theoretically, the scanning speed can be improved by three orders of magnitude. Thus the imaging velocity was significantly promoted. In terms of living body imaging, the high-speed-scan mode can effectively reduce the influence from the physiological activities to the living tissue. Therefore, this research has higher value of applying to the real-time imaging on material surface.

\section{Acknowledgements}

This work was sponsored by the Scientific Research Fund from Shaanxi Province Education Committee (No.06JK283) and supported in part by a grant from the National Natural Science Foundation of China (No.F051205). 


\section{References}

1. X.M. Liu, M.J. Cobb, X.D. Li: Optic Letters.2004, 29(1), P. 80-82.

2. S. Bourquin, V. Monterosso, P. Seitz, et al.: Optics Letters, 2006,25(2), P. 102-104.

3. M. Suekuni, Y. Teramura: J. Opt A: Pure Apple Phys,2005,38, P. 2519-2535.

4. D. Huang, E.A. Swanson, et al: Science,1991,254, P. 1178-1181

5. J.G. Wu, M. Conry, C.H. Gu: Optics Letters, 2015,40(9), P. 1265-1267.

6. G. Li, W. Shao, K.J. Wu, et al.: Proceedings of SPIE, 2012, 5916, P. 536-542.

7. P.H. Tomlins, R.K. Wang: J.Phys D: Appl Phys, 2005,38, P. 2519-2535.

8. A.F. Fercher, W. Drexler, C.K. Hitzenberger: Rep Prog Phys, 2013, 76, P. 239-303. 